\title{
Tree Path Majority Data Structures
}

Travis Gagie ${ }^{1}$

CeBiB - Center for Biotechnology and Bioengineering, Chile

School of Computer Science and Telecommunications, Diego Portales University, Chile

travis.gagie@gmail.com

\section{Meng $\mathbf{H e}^{2}$}

Faculty of Computer Science, Dalhousie University, Canada

mhe@cs.dal.ca

\section{Gonzalo Navarro ${ }^{3}$}

CeBiB - Center for Biotechnology and Bioengineering, Chile

IMFD - Millenium Institute for Foundational Research on Data, Chile

Dept. of Computer Science, University of Chile, Chile

gnavarro@dcc.uchile.cl

\begin{abstract}
We present the first solution to $\tau$-majorities on tree paths. Given a tree of $n$ nodes, each with a label from [1.. $\sigma]$, and a fixed threshold $0<\tau<1$, such a query gives two nodes $u$ and $v$ and asks for all the labels that appear more than $\tau \cdot\left|P_{u v}\right|$ times in the path $P_{u v}$ from $u$ to $v$, where $\left|P_{u v}\right|$ denotes the number of nodes in $P_{u v}$. Note that the answer to any query is of size up to $1 / \tau$. On a $w$-bit RAM, we obtain a linear-space data structure with $O\left((1 / \tau) \lg ^{*} n \lg \lg _{w} \sigma\right)$ query time. For any $\kappa>1$, we can also build a structure that uses $O\left(n \lg ^{[\kappa]} n\right)$ space, where $\lg ^{[\kappa]} n$ denotes the function that applies logarithm $\kappa$ times to $n$, and answers queries in time $O\left((1 / \tau) \lg \lg _{w} \sigma\right)$. The construction time of both structures is $O(n \lg n)$. We also describe two succinct-space solutions with the same query time of the linear-space structure. One uses $2 n H+4 n+o(n)(H+1)$ bits, where $H \leq \lg \sigma$ is the entropy of the label distribution, and can be built in $O(n \lg n)$ time. The other uses $n H+O(n)+o(n H)$ bits and is built in $O(n \lg n)$ time w.h.p.
\end{abstract}

2012 ACM Subject Classification Theory of computation $\rightarrow$ Data structures design and analysis

Keywords and phrases Majorities on Trees, Succinct data structures

Digital Object Identifier 10.4230/LIPIcs.ISAAC.2018.68

Related Version An extended version of the paper is available at https://arxiv.org/abs/1806. 01804.

\section{Introduction}

Finding frequent elements in subsets of a multiset is fundamental in data analysis and data mining $[12,10]$. When the sets have a certain structure, it is possible to preprocess the multiset to build data structures that efficiently find the frequent elements in any subset.

The best studied multiset structure is the sequence, where the subsets that can be queried are ranges (i.e., contiguous subsequences) of the sequence. Applications of this case include time sequences, linear-versioned structures, and one-dimensional models, for example. Data

1 Funded by FONDECYT grant 1171058, Chile.

2 Funded by NSERC, Canada.

3 Funded with basal funds FB0001, Conicyt, Chile, by Millenium Institute for Foundational Research on Data (IMFD), Chile, and by Fondecyt grant 1170048, Chile. 
structures for finding the mode (i.e., the most frequent element) in a range require time $O(\sqrt{n / \lg n})$, and it is unlikely that this can be done much better within reasonable extra space [8]. Instead, listing all the elements whose relative frequency in a range is over some fraction $\tau$ (called the $\tau$-majorities of the range) is feasible within linear space and $O(1 / \tau)$ time, which is worst-case optimal [1]. Mode and $\tau$-majority queries on higher-dimensional arrays have also been studied $[13,8]$.

In this paper we focus on finding frequent elements when the subsets that can be queried are the labels on paths from one given node to another in a labeled tree. For example, given a minimum spanning tree of a graph, we might be interested in frequent node types on the path between two nodes. Path mode or $\tau$-majority queries on multi-labeled trees could be useful when handling the tree of versions of a document or a piece of software, or a phylogenetic tree (which is essentially a tree of versions of a genome). If each node stores a list of the sections (i.e., chapters, modules, genes) on which its version differs from its parent's, then we can efficiently query which sections are changed most frequently between two given versions.

There has been little work previously on finding frequent elements on tree paths. Krizanc et al. [15] considered path mode queries, obtaining $O(\sqrt{n} \lg n)$ query time. This was recently improved by Durocher et al. [11], who obtained $O(\sqrt{n / w} \lg \lg n)$ time on a RAM machine of $w=\Omega(\lg n)$ bits. Like on the more special case of sequences, these times are not likely to improve much. No previous work has considered the problem of finding path $\tau$-majority queries, which is more tractable than finding the path mode. This is our focus.

We present the first data structures to support path $\tau$-majority queries on trees of $n$ nodes, with labels in $[1 . . \sigma]$, on a RAM machine. We first obtain a data structure using $O(n \lg n)$ space and $O\left((1 / \tau) \lg _{\lg } \sigma\right)$ time (Theorem 3). Building on this result, we reduce the space to $O(n)$ at the price of a very slight increase in the query time, $O\left((1 / \tau) \lg ^{*} n \lg \lg _{w} \sigma\right)$ (Theorem 6). We then show that the original query time can be obtained within very slightly superlinear space, $O\left(n \lg ^{[\kappa]} n\right)$ for any desired $\kappa>1$, where $\lg ^{[\kappa]} n$ denotes the function that applies logarithm $\kappa$ times to $n$ (Theorem 7). Finally, we show that our linear-space data structure can be further compressed, to either $2 n H+4 n+o(n)(H+1)$ bits or $n H+O(n)+o(n H)$ bits, where $H \leq \lg \sigma$ is the entropy of the distribution of the labels in $T$, while retaining the same query times of the linear-space data structure (Theorems 8 and 9). All our structures can be built in $O(n \lg n)$ deterministic time; only the latter one requires that time only w.h.p. We close with a brief discussion of directions for future research. In particular, we describe how to adapt our results to multi-labeled trees.

Durocher et al. [11] also considered queries that look for the least frequent elements and $\tau$-minorities on paths. In our extended version (https://arxiv.org/abs/1806.01804), we compress their data structure for $\tau$-minorities with only a very slight increase in query time.

\section{Preliminaries}

\subsection{Definitions}

We deal with rooted ordinal trees (or just trees) T. Further, our trees are labeled, that is, each node $u$ of $T$ has an integer label $\operatorname{label}(u) \in[1 . . \sigma]$. We assume that, if our main tree has $n$ nodes, then $\sigma=O(n)$ (we can always remap the labels to a range of size at most $n$ without altering the semantics of the queries of interest in this paper).

The path between nodes $u$ and $v$ in a tree $T$ is the (only) sequence of nodes $P_{u v}=\langle u=$ $\left.z_{1}, z_{2}, \ldots, z_{k-1}, z_{k}=v\right\rangle$ such that there is an edge in $T$ between each pair $z_{i}$ and $z_{i+1}$, for $1 \leq i<k$. The length of the path is $\left|P_{u v}\right|=k$, for example the length of the path $P_{u u}$ is 1 . Any path from $u$ to $v$ goes from $u$ to the lowest common ancestor of $u$ and $v$, and then from 
there it goes to $v$ (if $u$ is an ancestor of $v$ or vice versa, one of the two subpaths is empty).

Given a real number $0<\tau<1$, a $\tau$-majority of the path $P_{u v}$ is any label that appears (strictly) more than $\tau \cdot\left|P_{u v}\right|$ times among the labels of the nodes in $P_{u v}$. The path $\tau$-majority problem is, given $u$ and $v$, list all the $\tau$-majorities in the path $P_{u v}$. Note that there can be up to $\lfloor 1 / \tau\rfloor$ such $\tau$-majorities.

Our results hold in the RAM model of computation, assuming a computer word of $w=\Omega(\lg n)$ bits, supporting the standard operations.

Our logarithms are to the base 2 by default. By $\lg ^{[k]} n$ we mean the function that applies logarithm $k$ times to $n$, i.e., $\lg ^{[0]} n=n$ and $\lg ^{[k]} n=\lg \left(\lg ^{[k-1]} n\right)$. By $\lg ^{*} n$ we denote the iterated logarithm, i.e., the minimum $k$ such that $\lg ^{[k]} n \leq 1$.

\subsection{Sequence representations}

A bitvector $B[1 . . n]$ can be represented within $n+o(n)$ bits so that the following operations take constant time: $\operatorname{access}(B, i)$ returns $B[i], \operatorname{rank}_{b}(B, i)$ returns the number of times bit $b$ appears in $B[1 . . i]$, and select $(B, j)$ returns the position of the $j$ th occurrence of $b$ in $B[9]$. If $B$ has $m$ 1s, then it can be represented within $m \lg (n / m)+O(m)$ bits while retaining the same operation times [18]. Those structures can be built in linear time. Note the space is $o(n)$ bits if $m=o(n)$.

Analogous operations are defined on sequences $S[1 . . n]$ over alphabets [1.. $\sigma]$. For example, one can represent $S$ within $n H+o(n)(H+1)$ bits, where $H \leq \lg \sigma$ is the entropy of the distribution of symbols in $S$, so that rank takes time $O\left(\lg \lg _{w} \sigma\right)$, access takes time $O(1)$, and select takes any time in $\omega(1)[4$, Thm. 8]. The construction takes linear time. While this rank time is optimal, we can answer partial rank queries in $O(1)$ time, $\operatorname{prank}(S, i)=\operatorname{rank}_{S[i]}(S, i)$, by adding $O(n(1+\lg H))$ bits on top of a representation giving constant-time access [3, Sec. 3]. This construction requires linear randomized time.

\subsection{Range $\tau$-majorities on sequences}

A special version of the path $\tau$-majority queries on trees is range $\tau$-majority queries on sequences $S[1 . . n]$, which are much better studied. Given $i$ and $j$, the problem is to return all the distinct symbols that appear more than $\tau \cdot(j-i+1)$ times in $S[i . . j]$. The most recent result on this problem $[2,1]$ is a linear-space data structure, built in $O(n \lg n)$ time, that answers queries in the worst-case optimal time, $O(1 / \tau)$.

For our succinct representations, we also use a data structure [1, Thm. 6] that requires $n H+o(n)(H+1)$ bits, and can answer range $\tau$-majority queries in any time in $(1 / \tau) \cdot \omega(1)$. The structure is built on the sequence representation mentioned above [4, Thm. 8], and thus it includes its support for access, rank, and select queries on the sequence. To obtain the given times for $\tau$-majorities, the structure includes the support for partial rank queries [3, Sec. 3], and therefore its construction time is randomized. In this paper, however, it will be sufficient to obtain $O\left((1 / \tau) \lg \lg _{w} \sigma\right)$ time, and therefore we can replace their prank queries by general rank operations. These take time $O\left(\lg \lg _{w} \sigma\right)$ instead of $O(1)$, but can be built in linear time. ${ }^{4}$ Therefore, this slightly slower structure can also be built in $O(n \lg n)$ deterministic time.

\footnotetext{
${ }^{4}$ In fact, their structure [1] can be considerably simplified if one can spend the time of a general rank query per returned majority.
} 
When a set has no structure, we can find its $\tau$-majorities in linear time. Misra and Gries [16] proposed an optimal solution that computes all $\tau$-majorities using $O(n \lg (1 / \tau))$ comparisons. When implemented on a word RAM over an integer alphabet of size $\sigma$, the running time becomes $O(n)$ [10].

\subsection{Tree operations}

For tree nodes $u$ and $v$, we define the operations root (the tree root), parent $(u)$ (the parent of node $u$ ), $\operatorname{depth}(u)$ (the depth of node $u, 0$ being the depth of the root), preorder $(u)$ (the rank of $u$ in a preorder traversal of $T$ ), postorder $(u)$ (the rank of $u$ in a postorder traversal of $T$ ), subtreesize $(u$ ) (the number of nodes descending from $u$, including $u$ ), anc $(u, d$ ) (the ancestor of $u$ at depth $d$ ), and $l \mathrm{ca}(u, v)$ (the lowest common ancestor of $u$ and $v$ ). All those operations can be supported in constant time and linear space on a static tree after a linear-time preprocessing, trivially with the exceptions of anc [6] and lca [7].

A less classical query is $l$ abelanc $(u, \ell)$, which returns the nearest ancestor of $u$ (possibly $u$ itself) labeled $\ell$ (note that the label of $u$ needs not be $\ell$ ). If $u$ has no ancestor labeled $\ell$, labelanc $(u, \ell)$ returns null. This operation can be solved in time $O\left(\lg _{\lg } \lg _{w} \sigma\right)$ using linear space and preprocessing time $[14,21,11]$.

\subsection{Succinct tree representations}

A tree $T$ of $n$ nodes can be represented as a sequence $P[1 . .2 n]$ of parentheses (i.e., a bit sequence). In particular, we consider the balanced parentheses representation, where we traverse $T$ in depth-first order, writing an opening parenthesis when reaching a node and a closing one when leaving its subtree. A node is identified with the position $P[i]$ of its opening parenthesis. By using $2 n+o(n)$ bits, all the tree operations defined in Section 2.4 (except those on labels) can be supported in constant time [17].

This representation also supports access, rank and select on the bitvector of parentheses, and the operations close $(P, i)$ (the position of the parenthesis closing the one that opens at $P[i])$, open $(P, i)$ (the position of the parenthesis opening the one that closes at $P[i]$ ), and enclose $(P, i)$ (the position of the rightmost opening parenthesis whose corresponding parenthesis pair encloses $P[i]$; when $P$ represents a tree, this parenthesis represents the parent of the node that $P[i]$ corresponds to).

Labeled trees can be represented within $n H+2 n+o(n)(H+1)$ bits by adding the sequence $S[1 . . n]$ of the node labels in preorder, so that label $(i)=\operatorname{access}(S, \operatorname{preorder}(i))$.

\section{An $O(n \lg n)$-Space Solution}

In this section we design a data structure answering path $\tau$-majority queries on a tree of $n$ nodes using $O(n \lg n)$ space and $O\left((1 / \tau) \lg _{\lg } \sigma\right)$ time. This is the basis to obtain our final results.

We start by marking $O(\tau n)$ tree nodes, in a way that any node has a marked ancestor at distance $O(1 / \tau)$. A simple way to obtain these bounds is to mark every node whose height is $\geq\lceil 1 / \tau\rceil$ and whose depth is a multiple of $\lceil 1 / \tau\rceil$. Therefore, every marked node is the nearest marked ancestor of at least $\lceil 1 / \tau\rceil-1$ distinct non-marked nodes, which guarantees that there are $\leq \tau n$ marked nodes. On the other hand, any node is at distance at most $2\lceil 1 / \tau\rceil-1$ from its nearest marked ancestor. 
For each marked node $x$, we will consider prefixes $P_{i}(x)$ of the labels in the path from $x$ to the root, of length $1+2^{i}$, that is,

$$
P_{i}(x)=\left\langle\operatorname{label}(x), \operatorname{label}(\operatorname{parent}(x)), \operatorname{label}\left(\operatorname{parent}^{2}(x)\right), \ldots, \operatorname{label}\left(\operatorname{parent}^{2^{i}}(x)\right)\right\rangle
$$

(terminating the sequence at the root if we reach it). For each $0 \leq i \leq\lceil\lg \operatorname{depth}(x)\rceil$, we store $C_{i}(x)$, the set of $(\tau / 2)$-majorities in $P_{i}(x)$. Note that $\left|C_{i}(x)\right| \leq 2 / \tau$ for any $x$ and $i$.

By successive applications of the next lemma we have that, to find all the $\tau$-majorities in the path from $u$ to $v$, we can partition the path into several subpaths and then consider just the $\tau$-majorities in each subpath.

- Lemma 1. Let $u$ and $v$ be two tree nodes, and let $z$ be an intermediate node in the path. Then, a $\tau$-majority in the path from $u$ to $v$ is a $\tau$-majority in the path from $u$ to $z$ (including $z$ ) or a $\tau$-majority in the path from $z$ to $v$ (excluding $z$ ), or in both.

Proof. Let $d_{u z}$ be the distance from $u$ to $z$ (counting $z$ ) and $d_{z v}$ be the distance from $z$ to $v$ (not counting $z$ ). Then the path from $u$ to $v$ is of length $d=d_{u z}+d_{z v}$. If a label $\ell$ occurs at most $\tau \cdot d_{u z}$ times in the path from $u$ to $z$ and at most $\tau \cdot d_{z v}$ times in the path from $z$ to $v$, then it occurs at most $\tau\left(d_{u z}+d_{z v}\right)=\tau \cdot d$ times in the path from $u$ to $v$.

Let us now show that the candidates we record for marked nodes are sufficient to find path $\tau$-majorities towards their ancestors.

- Lemma 2. Let $x$ be a marked node. All the $\tau$-majorities in the path from $x$ to a proper ancestor $z$ are included in $C_{i}(x)$ for some suitable $i$.

Proof. Let $d_{x z}=\operatorname{depth}(x)-\operatorname{depth}(z)$ be the distance from $x$ to $z$ (i.e., the length of the path from $x$ to $z$ minus 1$)$. Let $i=\left\lceil\lg d_{x z}\right\rceil$. The path $P_{i}(x)$ contains all the nodes in an upward path of length $1+2^{i}$ starting at $x$, where $d_{x z} \leq 2^{i}<2 d_{x z}$. Therefore, $P_{i}(x)$ contains node $z$, but its length is $\left|P_{i}(x)\right|<1+2 d_{x z}$. Therefore, any $\tau$-majority in the path from $x$ to $z$ appears more than $\tau \cdot\left(1+d_{x z}\right)>(\tau / 2) \cdot\left(1+2 d_{x z}\right)>(\tau / 2) \cdot\left|P_{i}(x)\right|$ times, and thus it is a $(\tau / 2)$-majority recorded in $C_{i}(x)$.

\subsection{Queries}

With the properties above, we can find a candidate set of size $O(1 / \tau)$ for the path $\tau$-majority between arbitrary tree nodes $u$ and $v$. Let $z=1$ ca $(u, v)$. If $v \neq z$, let us also define $z^{\prime}=\operatorname{anc}(v, \operatorname{depth}(z)+1)$, that is, the child of $z$ in the path to $v$. The path is then split into at most four subpaths, each of which can be empty:

1. The nodes from $u$ to its nearest marked ancestor, $x$, not including $x$. If $x$ does not exist or is a proper ancestor of $z$, then this subpath contains the nodes from $u$ to $z$. The length of this path is less than $2\lceil 1 / \tau\rceil$ by the definition of marked nodes, and it is empty if $u=x$.

2. The nodes from $v$ to its nearest marked ancestor, $y$, not including $y$. If $y$ does not exist or is an ancestor of $z$, then this subpath contains the nodes from $v$ to $z^{\prime}$. The length of this path is again less than $2\lceil 1 / \tau\rceil$, and it is empty if $v=y$ or $v=z$.

3. The nodes from $x$ to $z$. This path exists only if $x$ exists and descends from $z$.

4. The nodes from $y$ to $z^{\prime}$. This path exists only if $y$ exists and descends from $z^{\prime}$.

By Lemma 1 , any $\tau$-majority in the path from $u$ to $v$ must be a $\tau$-majority in some of these four paths. For the paths 1 and 2, we consider all their up to $2\lceil 1 / \tau\rceil-1$ nodes as candidates. For the paths 3 and 4 , we use Lemma 2 to find suitable values $i$ and $j$ so that $C_{i}(x)$ and $C_{j}(y)$, both of size at most $2 / \tau$, contain all the possible $\tau$-majorities in those paths. In total, we obtain a set of at most $8 / \tau+O(1)$ candidates that contain all the $\tau$-majorities in the path from $u$ to $v$. 
To verify whether a candidate is indeed a $\tau$-majority, we follow the technique of Durocher et al. [11]. Every tree node $u$ will store count $(u)$, the number of times its label occurs in the path from $u$ to the root. We also make use of operation labelanc $(u, \ell)$. If $u$ has no ancestor labeled $\ell$, this operation returns null, and we define count $($ null $)=0$. Therefore, the number of times label $\ell$ occurs in the path from $u$ to an ancestor $z$ of $u$ (including $z$ ) can be computed as count (labelanc $(u, \ell))-\operatorname{count}(\operatorname{labelanc}(\operatorname{parent}(z), \ell))$. Each of our candidates can then be checked by counting their occurrences in the path from $u$ to $v$ using

$$
\begin{aligned}
& (\operatorname{count}(\operatorname{labelanc}(u, \ell))-\operatorname{count}(\operatorname{labelanc}(\operatorname{parent}(z), \ell))) \\
& +(\operatorname{count}(\operatorname{labelanc}(v, \ell))-\operatorname{count}(\operatorname{labelanc}(z, \ell))) .
\end{aligned}
$$

The time to perform query labelanc is $O\left(\lg \lg _{w} \sigma\right)$ using a linear-space data structure on the tree $[14,21,11]$, and therefore we find all the path $\tau$-majorities in time $O\left((1 / \tau) \lg \lg _{w} \sigma\right)$.

The space of our data structure is dominated by the $O(\lg n)$ candidate sets $C_{i}(x)$ we store for the marked nodes $x$. These amount to $O((1 / \tau) \lg n)$ space per marked node, of which there are $O(\tau n)$. Thus, we spend $O(n \lg n)$ space in total.

- Theorem 3. Let $T$ be a tree of $n$ nodes with labels in $[1 . . \sigma]$, and $0<\tau<1$. On a $R A M$ machine of $w$-bit words, we can build an $O(n \lg n)$ space data structure that answers path $\tau$-majority queries in time $O\left((1 / \tau) \lg \lg _{w} \sigma\right)$.

\subsection{Construction}

The construction of the data structure is easily carried out in linear time (including the fields count and the data structure to support labelanc [11]), except for the candidate sets $C_{i}(x)$ of the marked nodes $x$. We can compute the sets $C_{i}(x)$ for all $i$ in total time $O(\operatorname{depth}(x))$ using the linear-time algorithm of Misra and Gries [16] because we compute $(\tau / 2)$-majorities of doubling-length prefixes $P_{i}(x)$. This amounts to time $O(m t)$ on a tree of $t$ nodes and $m$ marked nodes. In our case, where $t=n$ and $m \leq \tau n$, this is $O\left(\tau n^{2}\right)$.

To reduce this time, we proceed as follows. First we build all the data structure components except the sets $C_{i}(x)$. We then decompose the tree into heavy paths [20] in linear time, and collect the labels along the heavy paths to form a set of sequences. On the sequences, we build in $O(t \lg t)$ time the range $\tau$-majority data structure $[2,1]$ that answers queries in time $O(1 / \tau)$. The prefix $P_{i}(x)$ for any marked node $x$ then spans $O(\lg t)$ sequence ranges, corresponding to the heavy paths intersected by $P_{i}(x)$. We can then compute $C_{i}(x)$ by collecting and checking the $O(1 / \tau)(\tau / 2)$-majorities from each of those $O(\lg t)$ ranges.

Let the path from $x$ to the root be formed by $O(\lg t)$ heavy path segments $\pi_{1}, \ldots, \pi_{k}$ We first compute the $O(1 / \tau)(\tau / 2)$-majority in the sequences corresponding to each prefix $\pi_{1}, \ldots, \pi_{k}$ : For each $\pi_{j}$, we (1) compute its $2 / \tau$ majorities on the corresponding sequence in time $O(1 / \tau),(2)$ add them to the set of $2 / \tau$ majorities already computed for $\pi_{1}, \ldots, \pi_{j-1}$, and (3) check the exact frequencies of all the $4 / \tau$ candidates in the path $\pi_{1}, \ldots, \pi_{j}$ in time $O\left((1 / \tau) \lg \lg _{w} \sigma\right)$, using the structures already computed on the tree. All the $(\tau / 2)$-majorities for $\pi_{1}, \ldots, \pi_{j}$ are then found.

Each path $P_{i}(x)$ is formed by some prefix $\pi_{1}, \ldots, \pi_{j}$ plus a prefix of $\pi_{j+1}$. We can then carry out a process similar to the one to compute the majorities of $\pi_{1}, \ldots, \pi_{j+1}$, but using only the proper prefix of $\pi_{j+1}$. The $O(\lg t)$ sets $C_{i}(x)$ are then computed in total time $O\left((1 / \tau) \lg t \lg \lg _{w} \sigma\right)$. Added over the $m$ marked nodes, we obtain $O\left((1 / \tau) m \lg t \lg \lg { }_{w} \sigma\right)$ construction time.

- Lemma 4. On a tree of $t$ nodes, $m$ of which are marked, all the candidate sets $C_{i}(x)$ can be built in time $O\left((1 / \tau) m \lg t \lg \lg _{w} \sigma\right)$. 
The construction time in our case, where $t=n$ and $m \leq \tau n$, is the following.

- Corollary 5. The data structure of Theorem 3 can be built in time $O\left(n \lg n \lg \lg _{w} \sigma\right)$.

\section{A Linear-Space (and a Near-Linear-Space) Solution}

We can reduce the space of our data structure by stratifying our tree. First, let us create a separate structure to handle unary paths, that is, formed by nodes with only one child. The labels of upward maximal unary paths are laid out in a sequence, and the sequences of the labels of all the unary paths in $T$ are concatenated into a single sequence, $S$, of length at most $n$. On this sequence we build the linear-space data structure that solves range $\tau$-majority queries in time $O(1 / \tau)[2,1]$. Each node in a unary path of $T$ points to its position in $S$. Each node also stores a pointer to its nearest branching ancestor (i.e., ancestor with more than one child).

The stratification then proceeds as follows. We say that a tree node is large if it has more than $(1 / \tau) \lg n$ descendant nodes; other nodes are small. Then the subset of the large nodes, which is closed by parent, induces a subtree $T^{\prime}$ of $T$ with the same root and containing at most $\tau n / \lg n$ leaves, because for each leaf in $T^{\prime}$ there are at least $(1 / \tau) \lg n-1$ distinct nodes of $T$ not in $T^{\prime}$. Further, $T-T^{\prime}$ is a forest of trees $\left\{F_{i}\right\}$, each of size at most $(1 / \tau) \lg n$.

We will use for $T^{\prime}$ a structure similar to the one of Section 3, with some changes to ensure linear space. Note that $T^{\prime}$ may have $\Theta(n)$ nodes, but since it has at most $\tau n / \lg n$ leaves, $T^{\prime}$ has only $O(\tau n / \lg n)$ branching nodes. We modify the marking scheme, so that we mark exactly the branching nodes in $T^{\prime}$. Spending $O((1 / \tau) \lg n)$ space of the candidate sets $C_{i}(x)$ over all branching nodes of $T^{\prime}$ adds up to $O(n)$ space.

The procedure to solve path $\tau$-majority queries on $T^{\prime}$ is then as follows. We split the path from $u$ to $v$ into four subpaths, exactly as in Section 3. The subpaths of type 1 and 2 can now be of arbitrary length, but they are unary, thus we obtain their $1 / \tau$ candidates in time $O(1 / \tau)$ from the corresponding range of $S$. Finally, we check all the $O(1 / \tau)$ candidates in time $O\left((1 / \tau) \lg \lg _{w} \sigma\right)$ as in Section 3 .

The nodes $u$ and $v$ may, however, belong to some small tree $F_{i}$, which is of size $O((1 / \tau) \lg n)$. We preprocess all those $F_{i}$ in a way analogous to $T$. From each $F_{i}$ we define $F_{i}^{\prime}$ as the subtree of $F_{i}$ induced by the (parent-closed) set of the nodes with more than $(1 / \tau) \lg \lg n$ descendants; thus $F_{i}^{\prime}$ has $O\left(\left|F_{i}\right| \tau / \lg \lg n\right)$ branching nodes, which are marked. We store the candidate sets $C_{i}(x)$ of their marked nodes $x$, considering only the nodes in $F_{i}^{\prime}$.

If the candidates were stored as in Section 3, they would require $O((1 / \tau) \lg \sigma)$ bits per marked node. Instead of storing the candidate labels $\ell$ directly, however, we will store $\operatorname{depth}(y)$, where $y$ is the nearest ancestor of $x$ with label $\ell$. We can then recover $\ell=l \operatorname{abel}(\operatorname{anc}(x, \operatorname{depth}(y)))$ in constant time. Since the depths in $F_{i}$ are also $O((1 / \tau) \lg n)$, we need only $O(\lg ((1 / \tau) \lg n))$ bits per candidate. Further, by sorting the candidates by their $\operatorname{depth}(y)$ value, we can encode only the differences between consecutive depths using $\gamma$-codes [5]. Encoding $k$ increasing numbers in $[1 . . m]$ with this method requires $O(k \lg (m / k))$ bits; therefore we can encode our $O(1 / \tau)$ candidates using $O((1 / \tau) \lg \lg n)$ bits in total. Added over all the $O(\lg n)$ values of $i,{ }^{5}$ the candidates $C_{i}(x)$ require $O((1 / \tau) \lg \lg n)$ words per marked (i.e., branching) node. Added over all the branching nodes of $F_{i}^{\prime}$, this amounts to $O\left(\left|F_{i}^{\prime}\right|\right)$ space. The other pointers of $F_{i}$, as well as node labels, can be represented normally, as they are $O(n)$ in total.

\footnotetext{
${ }^{5}$ The values of $i$ are also bounded by $O(\lg ((1 / \tau) \lg n))$, but the bound $O(\lg n)=O(w)$ is more useful this time.
} 
The small nodes left out from the trees $F_{i}$ form a forest of subtrees of size $O((1 / \tau) \lg \lg n)$ each. We can iterate this process $\kappa$ times, so that the smallest trees are of size $O\left((1 / \tau) \lg ^{[\kappa]} n\right)$. We build no candidates sets on the smallest trees. We say that $T^{\prime}$ is a subtree of level 1 , our $F_{i}^{\prime}$ are subtrees of level 2, and so on, until the smallest subtrees, which are of level $\kappa$. Every node in $T$ has a pointer to the root of the subtree where it belongs in the stratification.

The general process to solve a path $\tau$-majority query from $u$ to $v$ is then as follows. We compute $z=1 \mathrm{ca}(u, v)$ and split the path from $u$ to $z$ into $k-k^{\prime}+1$ subpaths, where $k^{\prime}$ and $k$ (note $k^{\prime} \leq k \leq \kappa$ ) are the levels of the subtree where $z$ and $u$ belong, respectively. Let $u_{i}$ be the root of the subtree of level $i$ that is an ancestor of $u$, except that we set $u_{k^{\prime}}=z$.

1. If $k=\kappa$, then $u$ belongs to one of the smallest subtrees. We then collect the $O\left((1 / \tau) \lg ^{[\kappa]} n\right)$ node weights in the path from $u$ to $u_{\kappa}$ one by one and include them in the set of candidates. Then we move to the parent of that root, setting $u \leftarrow \operatorname{parent}\left(u_{\kappa}\right)$ and $k \leftarrow \kappa-1$.

2. At levels $k^{\prime} \leq k<\kappa$, if $u$ is a branching node, we collect the $2 / \tau$ candidates from the corresponding set $C_{i}(u)$, where $i$ is sufficient to cover $u_{k}\left(C_{i}(u)\right.$ will not store candidates beyond the subtree root). We then set $u \leftarrow \operatorname{parent}\left(u_{k}\right)$ and $k \leftarrow k-1$.

3. At levels $k^{\prime} \leq k<\kappa$, if $u$ is not a branching node, let $x$ be lowest between parent $(z)$ and the nearest branching ancestor of $u$. Let also $p$ be the position of $u$ in $S$. Then we find the $1 / \tau \tau$-majorities in $S[p . . p+\operatorname{depth}(u)-\operatorname{depth}(x)-1]$ in time $O(1 / \tau)$. We then continue from $u \leftarrow x$ and $k \leftarrow k(x)$, where $k(x)$ is the level of the subtree where $x$ belongs. Note that $k(x)$ can be equal to $k$, but it can also be any other level $k^{\prime} \leq k(x)<k$.

4. We stop when $u=\operatorname{parent}(z)$.

A similar procedure is followed to collect the candidates from $v$ to $z^{\prime}$. In total, since each path has at most one case 2 and one case 3 per level $k$, we collect at most $4 \kappa$ candidate sets of size $O(1 / \tau)$, plus two of size $O\left((1 / \tau) \lg ^{[\kappa]} n\right)$. The total cost to verify all the candidates is then $O\left((1 / \tau)\left(\kappa+\lg ^{[\kappa]} n\right) \lg \lg _{w} \sigma\right)$. The data structure uses linear space for any choice of $\kappa$, whereas the optimal time is obtained by setting $\kappa=\lg ^{*} n$.

The construction time, using the technique of Lemma 4 in level 1, is $O\left(n \lg \lg _{w} \sigma\right)$, since $T^{\prime}$ has $t=O(n)$ nodes and $m=O(\tau n / \lg n)$ marked nodes. For higher levels, we use the basic quadratic method described in the first lines of Section 3.2: a subtree $F$ of level $k$ has $t=O\left((1 / \tau) \lg ^{[k-1]} n\right)$ nodes and $m=O\left(\tau t / \lg ^{[k]} n\right)$ marked nodes, so it is built in time $O(m t)$. There are $O\left(\tau n / \lg ^{[k-1]} n\right)$ trees of level $k$, which gives a total construction time of $O\left(n \lg ^{[k-1]} n / \lg ^{[k]} n\right)$ for all the nodes in level $k$. Added over all the levels $k>1$, this yields $O(n \lg n / \lg \lg n)$. Both times, for $k=1$ and $k>1$, are however dominated by the $O(n \lg n)$ time to build the range majority data structure on $S$.

- Theorem 6. Let $T$ be a tree of $n$ nodes with labels in $[1 . . \sigma]$, and $0<\tau<1$. On a RAM machine of $w$-bit words, we can build in $O(n \lg n)$ time an $O(n)$ space data structure that answers path $\tau$-majority queries in time $O\left((1 / \tau) \lg ^{*} n \lg \lg _{w} \sigma\right)$.

On the other hand, we can use any constant number $\kappa$ of levels, and build the data structure of Section 3 on the last one, so as to ensure query time $O(1 / \tau)$ in this level as well. We use, however, the compressed storage of the candidates used in this section. With this storage format, a candidate set $C_{i}(x)$ takes $O\left((1 / \tau) \lg ^{[k]} n\right)$ bits. Multiplying by $\lg n$ (the crude upper bound on the number of $i$ values), this becomes $O\left((1 / \tau) \lg ^{[\kappa]} n\right)$ words. Since the trees are of size $O\left((1 / \tau) \lg ^{[\kappa-1]} n\right)$ and the sampling rate used in Section 3 is $\tau$, this amounts to $O\left((1 / \tau) \lg ^{[\kappa-1]} n \lg ^{[\kappa]} n\right)$ space per tree. Multiplied by the $O\left(\tau n / \lg ^{[\kappa-1]} n\right)$ trees of level $\kappa$, the total space is $O\left(n \lg ^{[\kappa]} n\right)$. 
The construction time of the candidate sets in the last level, using the basic quadratic construction, is $O(m t)=O\left((1 / \tau)\left(\lg ^{[\kappa-1]} n\right)^{2}\right)$, because $t=O\left((1 / \tau) \lg ^{[\kappa-1]} n\right)$ and $m=\tau t$ according to the sampling used in Section 3. Multiplying by the $O\left(\tau n / \lg ^{[\kappa-1]} n\right)$ trees of level $\kappa$, the total construction time for this last level is $O\left(n \lg ^{[\kappa-1]} n\right)$, again dominated by the time to build the range majority data structures if $\kappa>1$. This yields the next result.

- Theorem 7. Let $T$ be a tree of $n$ nodes with labels in $[1 . . \sigma]$, and $0<\tau<1$. On a RAM machine of $w$-bit words, for any constant $\kappa>1$, we can build in $O(n \lg n)$ time an $O\left(n \lg ^{[\kappa]} n\right)$ space data structure that answers path $\tau$-majority queries in time $O\left((1 / \tau) \lg \lg _{w} \sigma\right)$.

\section{A Succinct Space Solution}

The way to obtain a succinct space structure from Theorem 6 is to increase the thresholds that define the large nodes in Section 4. In level 1, we now define the large nodes as those whose subtree size is larger than $(1 / \tau)(\lg n)^{3}$; in level 2 , larger than $(1 / \tau)(\lg \lg n)^{3}$; and in general in level $k$ as those with subtree size larger than $(1 / \tau)\left(\lg ^{[k]} n\right)^{3}$. This makes the space of all the $C_{i}(x)$ structures to be $o(n)$ bits. The price is that the traversal of the smallest trees now produces $O\left((1 / \tau)\left(\lg ^{[\kappa]} n\right)^{3}\right)$ candidates, but this is easily sorted out by using $\kappa+1$ levels, since $\left(\lg ^{[\kappa+1]} n\right)^{3}=o\left(\lg ^{[\kappa]} n\right)$. To obtain succinct space, we will need that there are $o(n)$ subtrees of the smallest size, but that we find only $O\left((1 / \tau) \lg ^{*} n\right)$ candidates in total. Thus we set $\kappa=\lg ^{*} n-\lg ^{* *} n$, so that there are $O(\kappa)=O\left(\lg ^{*} n\right)$ levels, and the last-level subtrees are of size $O\left((1 / \tau)\left(\lg ^{[\kappa+1]} n\right)^{3}\right)=o\left((1 / \tau) \lg ^{\left[\lg ^{*} n-\lg ^{* *} n\right]} n\right)=o\left((1 / \tau) \lg ^{*} n\right)$. Still, there are $O\left(\tau n /\left(\lg ^{[\kappa+1]} n\right)^{3}\right)=O\left(\tau n /\left(\lg ^{\left[\lg ^{*} n-\lg ^{* *} n+1\right]} n\right)^{3}\right)=O\left(\tau n /\left(\lg \lg ^{*} n\right)^{3}\right)=o(n)$ subtrees in the last level.

The topology of the whole tree $T$ can be represented using balanced parentheses in $2 n+o(n)$ bits, supporting in constant time all the standard tree traversal operations we use [17]. We assume that opening and closing parentheses are represented with $1 \mathrm{~s}$ and $0 \mathrm{~s}$ in $P$, respectively. Let us now focus on the less standard operations needed.

\subsection{Counting labels in paths}

In Section 3, we count the number of times a label $\ell$ occurs in the path from $u$ to the root by means of a query labelanc and by storing count fields in the nodes. In Section 4 , we use in addition a string $S$ to support range majority queries on the unary paths.

To solve labelanc queries, we use the representation of Durocher et al. [11, Lem. 7], which uses $n H+2 n+o(n)(H+1)$ bits in addition to the $2 n+o(n)$ bits of the tree topology. This representation includes a string $S[1 . . n]$ where all the labels of $T$ are written in preorder; any implementation of $S$ supporting access, rank, and select in time $O\left(\lg \lg _{w} \sigma\right)$ can be used (e.g., [4]). This string can also play the role of the one we call $S$ in Section 4: the labels of unary paths are contiguous in $S$, and any node $v$ can access its label from $S$ [preorder $(v)]$.

On top of this string we must also answer range $\tau$-majority queries in time $O\left((1 / \tau) \lg \lg _{w} \sigma\right)$. We can use the slow variant of the succinct structure described in Section 2.3, which requires only $o(n)(H+1)$ additional bits and also supports access in $O(1)$ time and rank and select in time $O\left(\lg _{\lg _{w}} \sigma\right)$. This variant of the structure is built in $O(n \lg n)$ time.

In addition to supporting operation labelanc, we need to store or compute the count fields. Durocher et al. [11] also require this field, but find no succinct way to represent it. We now show a way to obtain this value within succinct space. 
The sequence $S$ lists the labels of $T$ in preorder, that is, aligned with the opening parentheses of $P$. Assume we have another sequence $S^{\prime}[1 . . n]$ where the labels of $T$ are listed in postorder (i.e., aligned with the closing parentheses of $P$ ). Since the opened parentheses not yet closed in $P[1 . i]$ are precisely node $i$ and its ancestors, we can compute the number of times a label $\ell$ appears in the path from $P[i]$ to the root as $\operatorname{rank}_{\ell}\left(S, \operatorname{rank}_{1}(P, i)\right)-$ $\operatorname{rank}_{\ell}\left(S^{\prime}, \operatorname{rank}_{0}(P, i)\right)$.

Therefore, we can support this operation with $n H+o(n)(H+1)$ additional bits. Note that, with this representation, we do not need the operation labelanc, since we do not need that $P[i]$ itself is labeled $\ell$.

If we do use operation labelanc, however, we can ensure that $P[i]$ is labeled $\ell$, and another solution is possible based on partial rank queries. Let $o=\operatorname{rank}_{\ell}\left(S, \operatorname{rank}_{1}(P, i)\right)$ and $c=\operatorname{rank}_{\ell}\left(S^{\prime}, \operatorname{rank}_{0}(P, i)\right)$ be the numbers of opening and closing parentheses up to $P[i]$, so that we want to compute $o-c$. Since $P[i]$ is labeled $\ell$, it holds that $\left.S\left[\operatorname{rank}_{1}(P, i)\right)\right]=\ell$, and thus $o=\operatorname{prank}\left(S, \operatorname{rank}_{1}(P, i)\right)$. To compute $c$, we do not store $S^{\prime}$, but rather $S^{\prime \prime}[1 . .2 n]$, so that $S^{\prime \prime}[i]$ is the label of the node whose opening or closing parenthesis is at $P[i]$ (i.e., $S^{\prime \prime}$ is formed by interleaving $S$ and $\left.S^{\prime}\right)$. Then, $\operatorname{prank}\left(S^{\prime \prime}, i\right)=o+c$; therefore the answer we seek is $o-c=2 \cdot \operatorname{prank}\left(S, \operatorname{rank}_{1}(P, i)\right)-\operatorname{prank}\left(S^{\prime \prime}, i\right)$.

We use the structure for constant-time partial rank queries [3, Sec. 3] that requires $O(n)+o(n H)$ bits on top of a sequence that can be accessed in $O(1)$ time. We can build it on $S$ and also on $S^{\prime \prime}$, though we do not explicitly represent $S^{\prime \prime}$ : any access to $S^{\prime \prime}$ is simulated in constant time with $S^{\prime \prime}[i]=S\left[\operatorname{rank}_{1}(P, i)\right]$ if $P[i]=1$, and $S^{\prime \prime}[i]=S\left[\operatorname{rank}_{1}(P, \operatorname{open}(P, i))\right]$ otherwise. This partial rank structure is built in $O(n)$ randomized time and in $O(n \lg n)$ time w.h.p. ${ }^{6}$

\subsection{Other data structures}

The other fields stored at tree nodes, which we must now compute within succinct space, are the following:

Pointers to candidate sets $\boldsymbol{C}_{\boldsymbol{i}}(\boldsymbol{x})$. All the branching nodes in all subtrees except those of level $\kappa+1$ are marked, and there are $O\left(n /\left(\lg ^{[\kappa+1]} n\right)^{3}\right)=o(n)$ such nodes. We can then mark their preorder ranks with $1 \mathrm{~s}$ in a bitvector $M[1 . . n]$. Since $M$ has $o(n) 1 \mathrm{~s}$, it can be represented within $o(n)$ bits [18] while supporting constant-time rank and select operations. We can then find out when a node $i$ is marked (iff $M[\operatorname{preorder}(i)]=1$ ), and if it is, its rank among all the marked nodes, $r=\operatorname{rank}_{1}(M$, preorder $(i))$. The $C_{i}(x)$ sets of all the marked nodes $x$ of any level can be written down in a contiguous memory area of total size $o(n)$ bits, sorted by the preorder rank of $x$. A bitvector $C$ of length $o(n)$ marks the starting position of each new node $x$ in this memory area. Then the area for marked node $i$ starts at $p=\operatorname{select}_{1}(C, r)$. A second bitvector $D$ can mark the starting position of each $C_{j}(x)$ in the memory area of each node $x$, and thus we access the specific set $C_{j}(x)$ from position $\operatorname{select}_{1}\left(D, \operatorname{rank}_{1}(D, p-1)+j\right)$.

Pointers to subtree roots. We store an additional bitvector $B[1 . .2 n]$, parallel to the parentheses bitvector $P[1 . .2 n]$. In $B$, we mark with 1 s the positions of the opening and closing parentheses that are roots of subtrees of any level. As there are $O\left(n /\left(\lg ^{[\kappa+1]} n\right)^{3}\right)=o(n)$ such nodes, $B$ can be represented within $o(n)$ bits while supporting constant-time rank and

${ }^{6}$ It involves building perfect hash functions, which succeeds with constant probability $p$ in time $O(n)$. Repeating $c \lg n$ times, the failure probability is $1-O\left(1 / n^{c / \lg (1 / p)}\right)$. 
select operations. We also store the sequence of $o(n)$ parentheses $P^{\prime}$ corresponding to those in $P$ marked with a 1 in $B$. Then the nearest subtree root containing node $P[i]$ is obtained by finding the nearest position to the left marked in $B, r=\operatorname{rank}_{1}(B, i)$ and $j=\operatorname{select}_{1}(B, r)$, and then considering the corresponding node $P^{\prime}[r]$. If it is an opening parenthesis, then the nearest subtree root is the node whose parenthesis opens in $P[j]$. Otherwise, it is the one opening at $P\left[j^{\prime}\right]$, where $j^{\prime}=\operatorname{select}_{1}\left(B, \operatorname{enclose}\left(P^{\prime}, \operatorname{open}\left(P^{\prime}, r\right)\right)\right)($ see $[19$, Sec. 4.1]).

Finding the nearest branching ancestor. A unary path looks like a sequence of opening parentheses followed by a sequence of closing parentheses. The nearest branching ancestor of $P[i]$ can then be obtained in constant time by finding the nearest closing parenthesis to the left, $l=\operatorname{select}_{0}\left(\operatorname{rank}_{0}(P, i)\right)$, and the nearest opening parenthesis to the right, $r=\operatorname{select}_{1}\left(\operatorname{rank}_{1}(\operatorname{close}(P, i))+1\right)$. Then the answer is the larger between enclose $(P$, open $(P, l))$ and enclose $(P, r)$.

Determining the subtree level of a node. Since we can compute $s=$ subtreesize $(i)$ of a node $P[i]$ in constant time, we can determine the corresponding level: if $s>(1 / \tau) \lg ^{3} n$, it is level 1. Otherwise, we look up $\tau \cdot s$ in a precomputed table of size $O\left(\lg ^{3} n\right)$ that stores the level corresponding to each possible size.

Therefore, depending on whether we represent both $S$ and $S^{\prime}$ or use partial rank structures, we obtain two results within succinct space.

- Theorem 8. Let $T$ be a tree of $n$ nodes with labels in [1.. $\sigma]$, and $0<\tau<1$. On a RAM machine of $w$-bit words, we can build in $O(n \lg n)$ time a data structure using $2 n H+4 n+o(n)(H+1)$ bits, where $H \leq \lg \sigma$ is the entropy of the distribution of the node labels, that answers path $\tau$-majority queries in time $O\left((1 / \tau) \lg ^{*} n \lg \lg _{w} \sigma\right)$.

- Theorem 9. Let $T$ be a tree of $n$ nodes with labels in $[1 . . \sigma]$, and $0<\tau<1$. On a $R A M$ machine of $w$-bit words, we can build in $O(n \lg n)$ time (w.h.p.) a data structure using $n H+O(n)+o(n H)$ bits, where $H \leq \lg \sigma$ is the entropy of the distribution of the node labels, that answers path $\tau$-majority queries in time $O\left((1 / \tau) \lg ^{*} n \lg \lg _{w} \sigma\right)$.

We note that, within this space, all the typical tree navigation functionality, as well as access to labels, is supported.

\section{Conclusions}

We have presented the first data structures that can efficiently find the $\tau$-majorities on the path between any two given nodes in a tree. Our data structures use linear or near-linear space, and even succinct space, whereas our query times are close to optimal, by a factor near log-logarithmic.

As mentioned in the Introduction, many applications of these results require that the trees are multi-labeled, that is, each node holds several labels. We can easily accommodate multi-labeled trees $T$ in our data structure, by building a new tree $T^{\prime}$ where each node $u$ of $T$ with $m(u)$ labels $\ell_{1}, \ldots, \ell_{m(u)}$ is replaced by an upward path of nodes $u_{1}, \ldots, u_{m(u)}$, each $u_{i}$ holding the label $\ell_{i}$ and being the only child of $u_{i+1}$ (and $u_{m(u)}$ being a child of $v_{1}$, where $v$ is the parent of $u$ in $T$ ). Path queries from $u$ to $v$ in $T$ are then transformed into path queries from $u_{1}$ to $v_{1}$ in $T^{\prime}$, except when $u(v)$ is an ancestor of $v(u)$, in which case we replace $u(v)$ by $u_{u(m)}\left(v_{m(v)}\right)$ in the query. All our complexities then hold on $T^{\prime}$, which is of size $n=\left|T^{\prime}\right|=\sum_{u \in T} m(u)$. 
Our query time for path $\tau$-majorities in linear space, $O\left((1 / \tau) \lg ^{*} n \lg \lg _{w} \sigma\right)$, is over the optimal time $O(1 / \tau)$ that can be obtained for range $\tau$-majorities on sequences [1]. It is open whether we can obtain optimal time on trees within linear or near-linear space. Other interesting research problems are solving $\tau^{\prime}$-majority queries for any $\tau^{\prime} \geq \tau$ given at query time, in time proportional to $1 / \tau^{\prime}$ instead of $1 / \tau$, and to support insertions and deletions of nodes in $T$. Similar questions can be posed for $\tau$-minorities, where the $O\left((1 / \tau) \lg \lg _{w} \sigma\right)$ query time of our linear-space solutions is also over the time $O(1 / \tau)$ achievable on sequences [1].

\section{References}

1 D. Belazzougui, T. Gagie, J. I. Munro, G. Navarro, and Y. Nekrich. Range Majorities and Minorities in Arrays. CoRR, abs/1606.04495, 2016. arXiv:1606.04495.

2 D. Belazzougui, T. Gagie, and G. Navarro. Better Space Bounds for Parameterized Range Majority and Minority. In Proc. 12th WADS, LNCS 8037, pages 121-132, 2013.

3 D. Belazzougui and G. Navarro. Alphabet-Independent Compressed Text Indexing. ACM Trans. Alg., 10(4):article 23, 2014.

4 D. Belazzougui and G. Navarro. Optimal Lower and Upper Bounds for Representing Sequences. ACM Trans. Alg., 11(4):article 31, 2015.

5 T. C. Bell, J. Cleary, and I. H. Witten. Text Compression. Prentice Hall, 1990.

6 M. Bender and M. Farach-Colton. The level ancestor problem simplified. Theor. Comp. Sci., 321(1):5-12, 2004.

7 M. A. Bender, M. Farach-Colton, G. Pemmasani, S. Skiena, and P. Sumazin. Lowest common ancestors in trees and directed acyclic graphs. J. Algorithms, 57(2):75-94, 2005.

8 T. M. Chan, S. Durocher, K. G. Larsen, J. Morrison, and B. T. Wilkinson. Linear-Space Data Structures for Range Mode Query in Arrays. Theor. Comp. Syst., 55(4):719-741, 2014.

9 D. R. Clark. Compact PAT Trees. PhD thesis, University of Waterloo, Canada, 1996.

10 E. D. Demaine, A. López-Ortiz, and J. I. Munro. Frequency Estimation of Internet Packet Streams with Limited Space. In Proc. 10th ESA, pages 348-360, 2002.

11 S. Durocher, R. Shah, M. Skala, and S. V. Thankachan. Linear-space data structures for range frequency queries on arrays and trees. Algorithmica, 74(1):344-366, 2016.

12 M. Fang, N. Shivakumar, H. Garcia-Molina, R. Motwani, and J. D. Ullman. Computing Iceberg Queries Efficiently. In Proc. 24th VLDB, pages 299-310, 1998.

13 T. Gagie, M. He, J. I. Munro, and P. K. Nicholson. Finding Frequent Elements in Compressed 2D Arrays and Strings. In Proc. 18th SPIRE, pages 295-300, 2011.

14 M. He, J. I. Munro, and G. Zhou. A Framework for Succinct Labeled Ordinal Trees over Large Alphabets. Algorithmica, 70(4):696-717, 2014.

15 D. Krizanc, P. Morin, and M. H. M. Smid. Range mode and range median queries on lists and trees. Nordic J. Comp., 12(1):1-17, 2005.

16 J. Misra and D. Gries. Finding Repeated Elements. Sci. Comp. Prog., 2(2):143-152, 1982.

17 G. Navarro and K. Sadakane. Fully-Functional Static and Dynamic Succinct Trees. ACM Trans. Alg., 10(3):article 16, 2014.

18 R. Raman, V. Raman, and S. S. Rao. Succinct indexable dictionaries with applications to encoding $k$-ary trees, prefix sums and multisets. ACM Trans. Alg., 3(4):article 43, 2007.

19 L. Russo, G. Navarro, and A. Oliveira. Fully-Compressed Suffix Trees. ACM Trans. Alg., 7(4):article 53, 2011.

20 D. Sleator and R. E. Tarjan. A data structure for dynamic trees. J. Comp. Sys. Sci., 26(3):362-391, 1983.

21 D. Tsur. Succinct representation of labeled trees. Theor. Comp. Sci., 562:320-329, 2014. 